\title{
Delayed anastomotic leakage during treatment with ramucirumab: A case report
}

\author{
Yusuke Takahashi*, Akira Kobayashi and Hitoshi Seki \\ Department of Digestive Surgery, Nagano Municipal Hospital, Nagano, 381-8551, Japan
}

\begin{abstract}
While adverse gastrointestinal events related to chemotherapy are known to occur, delayed anastomotic leakage during chemotherapy with concurrent ramucirumab treatment has never been reported. We describe the clinical course of a patient who experienced delayed anastomotic leakage during 5-fluorouracil, leucovorin, and irinotecan (FOLFIRI) and ramucirumab treatments, which was ultimately fatal. The patient was a 78-year-old man who underwent laparoscopic right hemicolectomy with regional lymphadenectomy for advanced ascending colon cancer that was symptomatic and accompanied by unresectable and multiple liver metastases. Anastomosis was performed using a stapler (a functional end-to-end anastomosis technique); the postoperative course was uneventful. One-month post-surgery, the patient received five courses of chemotherapy, which included 5-fluorouracil, leucovorin, and oxaliplatin (FOLFOX) with bevacizumab as the first-line treatment and FOLFIRI with ramucirumab as the second-line treatment due to the progression of liver metastases observed on contrast-enhanced computed tomography. During the sixth course of ramucirumab and FOLFIRI, the patient reported right lower abdominal pain. Butylscopolamine was administered because the abdominal pain was considered a side effect of irinotecan, and his symptoms slightly improved. That night, he presented to our emergency room with sustained abdominal pain. Abdominal computed tomography showed intra-abdominal free air and anastomotic leakage at the ileo-colonic anastomosis from his previous surgery, without any peritoneal dissemination or local recurrence. Emergency surgery including resection of the leaked anastomosis and ileostomy was performed. Postoperative ileus was observed, and leucopenia (neutropenia) occurred from postoperative day 7. Severe pneumonia and respiratory failure also occurred. The patient died on postoperative day 11. We believe that our patient's delayed anastomotic leakage (6 months post-initial surgery) was a novel adverse event related to ramucirumab use. Although abdominal pain may occur after irinotecan administration, clinicians should be aware of this potentially fatal side effect of ramucirumab to facilitate rapid treatment.
\end{abstract}

\section{Background}

Anti-vascular endothelial growth factor (anti-VEGF) agents such as bevacizumab, ramucirumab, and aflibercept are combined with 5-fluorouracil (5-FU)-based chemotherapy to treat metastatic colorectal cancer [1-3]. These agents are occasionally associated with gastrointestinal perforation; however, delayed anastomotic leakage following ramucirumab administration has not been previously reported [4].

Here, we report about a patient who experienced delayed anastomotic leakage that occurred six months after right hemicolectomy while undergoing chemotherapy with ramucirumab.

\section{Case presentation}

A 78-year-old man with no relevant comorbidities underwent laparoscopic right hemicolectomy for advanced symptomatic ascending colon cancer with unresectable liver metastasis (T3N1M1a Stage IVa in the UICC classification 8th edition) (Figure 1). Anastomosis of the remnant ileum and transverse colon was performed using a stapler (EES liner cutter, Ethicon, Cincinnati, $\mathrm{OH}$ ) via a functional end-toend anastomosis technique. No complications related to the surgery were observed. One-month post-surgery, the patient received 5-FU, leucovorin, and oxaliplatin (FOLFOX) plus bevacizumab as first-line therapy for unresectable liver metastases. After five cycles of FOLFOX plus bevacizumab, multiple liver metastases were enlarged, and no other recurrence was seen on contrast-enhanced computed tomography (CT). Therefore, the patient received 5-FU, leucovorin, and irinotecan (FOLFIRI) plus ramucirumab as second-line therapy.
During the sixth cycle of FOLFIRI plus ramucirumab, at 6 months post-surgery, the patient reported abdominal pain. Localized right lower tenderness was observed on physical examination, and no imaging tests were performed. Butylscopolamine was administered intravenously because irinotecan-induced cholinergic syndrome was suspected. His symptoms slightly improved, and the patient went home. That night, he presented to our emergency room with sustained abdominal pain. Rebound tenderness and muscular defense were observed. Contrastenhanced abdominal CT showed free air (Figure 2). An emergency surgery was performed. Anastomotic leakage was detected at the ileocolonic anastomosis from the previous surgery, while no peritoneal dissemination or local recurrence was observed (Figure 3). Resection of the leaked anastomosis and ileostomy were performed. The cause of the delayed leakage was unclear in pathological examination.

A postoperative ileus was observed but was not related to the resection of the leaked anastomosis or ileostomy. Febrile neutropenia was also present beginning on postoperative day (POD) 7 , with a minimum neutrophil count of $22 / \mu \mathrm{l}$, and acute respiratory distress

*Correspondence to: Yusuke Takahashi, MD Oncologist, Department of Digestive Surgery, Nagano Municipal Hospital, 1-1333 Tomitake, Nagano City, 381-8551, Japan, Tel: +81-26-295-1199, Fax: +81-26-295-1148, E-mail: yusuke_ takahashi@hospital.nagano.nagano.jp

Key words: anastomotic leak, drug therapy, ramucirumab

Received: February 10, 2021; Accepted: February 24, 2021; Published: March 01,2021 
syndrome occurred on POD 9 (Figure 4). The patient died on POD 11 despite multidisciplinary treatment.

\section{Discussion}

Though extremely rare, gastrointestinal perforation related to the use of anti-VEGF agents has been reported in the clinical trials of these drugs [1-4]. The use of anti-VEGF agents is also a risk factor for perforation in patients with metal stents placed to treat obstructive colon cancer [5]. According to the National Comprehensive Cancer Network Guidelines, an interval of at least 6 weeks is necessary between the final bevacizumab treatment and surgery [6]. This is likely due to impaired wound healing, which can lead to wound dehiscence and anastomotic leakage. In addition to gastrointestinal perforation, bleeding and thrombotic events during treatment with anti-VEGF agents have been reported, caused by the pharmacological effects of anti-VEGF agents [7].

Various perioperative risks and adverse events have been described during or after anti-VEGF agent use; however, to the best of our knowledge, a case of delayed anastomotic leakage following colectomy has not been reported. In our case, the cause of delayed anastomotic leakage was pathologically unclear. In this case, the

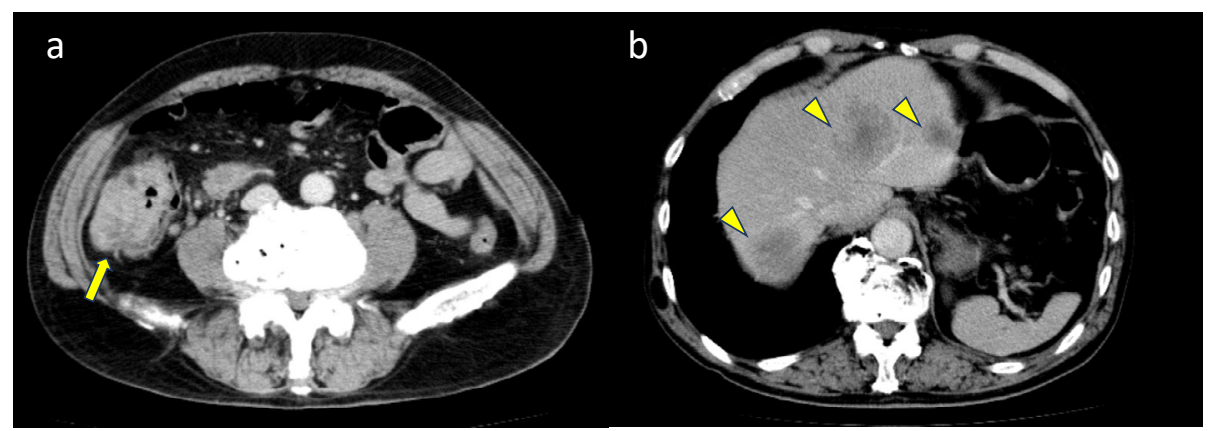

Figure 1. Contrast-enhanced computed tomography before surgery for primary colon cancer (A) Arrow: primary ascending colon cancer (B) Arrowhead: multiple liver metastases from colon cancer

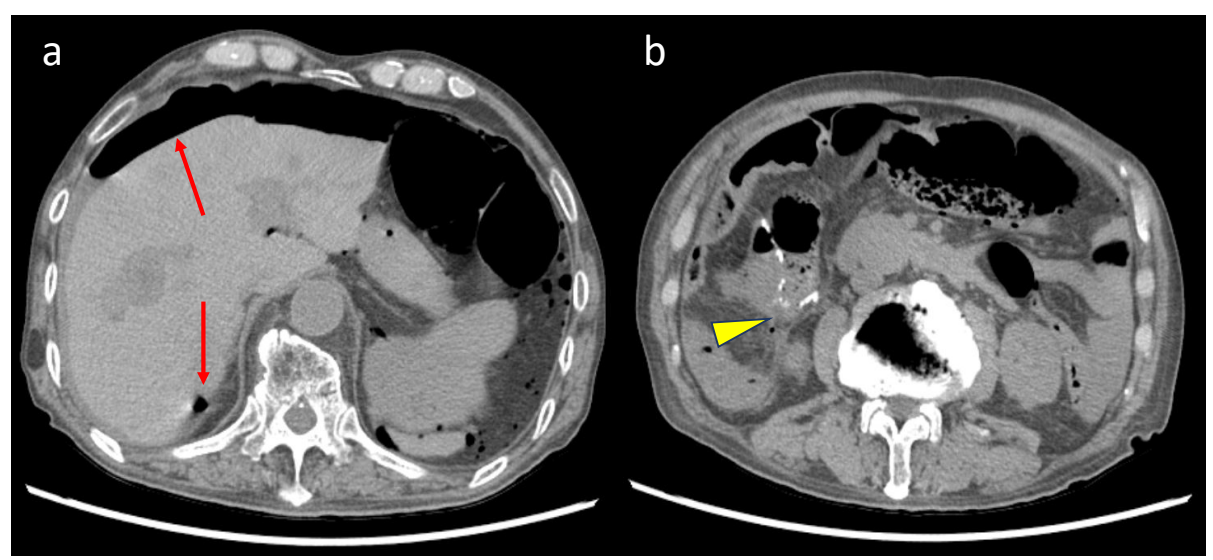

Figure 2. Contrast-enhanced computed tomography of the delayed anastomotic leakage (A) Arrows: intra-abdominal free air (B) Arrowhead: free air beside the anastomosis performed after right hemicolectomy

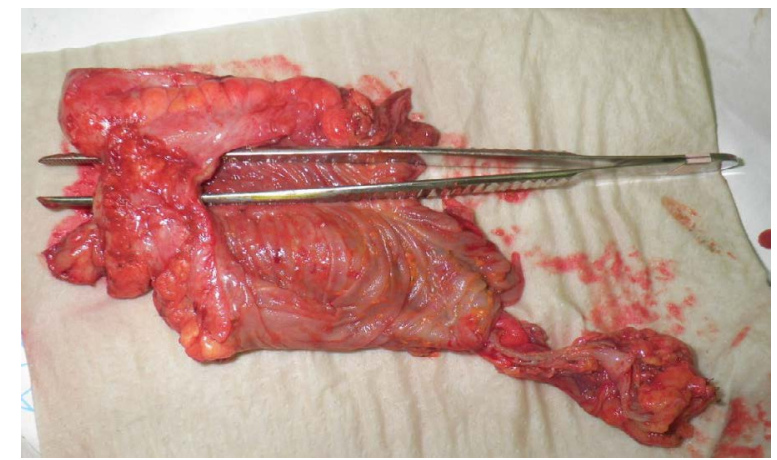

Figure 3. Resected specimen, The forceps are inserted in the previous surgical anastomosis, at the point of delayed anastomotic leakage 


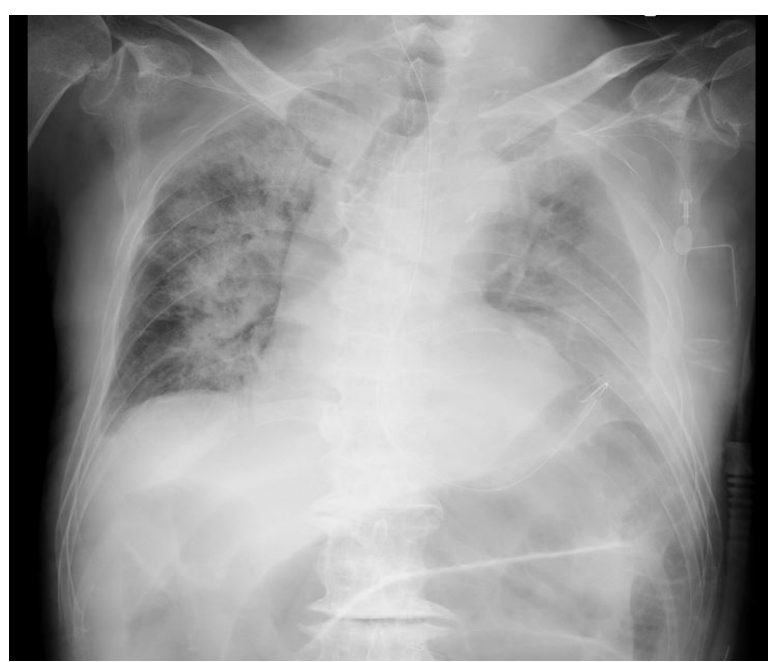

Figure 4. Chest X-ray showing acute respiratory distress syndrome. Bilateral infiltration was observed on postoperative day 9

ileum and remnant colon were connected by a functional end-toend anastomosis with a stapler. The staple line may be potentially susceptible to ischemia, since anastomotic leakage usually occurs from the staple line. We suspect that this event was caused by ramucirumab use. Ramucirumab-induced impairments in the blood supply may lead to delayed anastomotic leakage from the staple line, as shown in Figure 3. In contrast, it is worth noting that chemotherapy itself did not appear to influence delayed anastomotic leakage.

The patient's complaint of abdominal pain was initially thought to be due to irinotecan-induced cholinergic syndrome. In clinical trials using FOLFIRI with ramucirumab, the frequency of abdominal pain was $26 \%$, while that of gastrointestinal perforation was less than $3 \%$ [2]. Butylscopolamine was administered to the patient because it has been reported to be effective for treating cholinergic syndrome [8]. The patient's symptoms were not completely resolved, and he returned to our hospital with sustained abdominal pain. The patient was diagnosed with panperitonitis, but the clinical course was unsuccessful despite emergency surgery. Although gastrointestinal perforation-including delayed anastomotic leakage is extremely rare, prompt diagnosis and appropriate management may enable successful treatment.

\section{Conclusions}

Delayed anastomotic leakage following colectomy can occur with anti-VEGF agent use even several months after surgery. Gastrointestinal perforation should be distinguished from irinotecaninduced cholinergic syndrome during treatment with FOLFIRI with ramucirumab because delayed diagnosis and treatment may lead to devastating clinical outcomes.

\section{Authors' contributions}

YT produced the study concept and design and wrote the case report. HS participated in the treatment of the patient and drafted the manuscript. HS and AK critically revised the manuscript. All authors read and approved the final manuscript.

\section{Acknowledgments}

We would like to thank Editage (www.editage.com) for their writing support on the Abstract.

\section{Funding}

This research received no external funding.

\section{Competing interests}

The authors declare that they have no competing interests.

\section{Declarations}

Ethics approval and consent to participate: Our institutional review board waived the requirement of ethical approval for this case report.

\section{Consent for publication}

We obtained written consent from the patient's family to publish this case report.

\section{Availability of data and material}

The data supporting the findings of this study are available within the article.

\section{References}

1. Saltz LB, Clarke S, Díaz-Rubio E, Scheithauer W, Figer A, et al. (2008) Bevacizumab in combination with oxaliplatin-based chemotherapy as first-line therapy in metastatic colorectal cancer: a randomized phase III study. Journal of Clinical Oncology 26: 2013-2019. [Crossref]

2. Tabernero J, Yoshino T, Cohn AL, Obermannova R, Bodoky G, et al. (2015) Ramucirumab versus placebo in combination with second-line FOLFIRI in patients with metastatic colorectal carcinoma that progressed during or after first-line therapy with bevacizumab, oxaliplatin, and a fluoropyrimidine (RAISE): a randomised, doubleblind, multicentre, phase 3 study. The Lancet Oncology 16: 499-508. [Crossref]

3. Van Cutsem E, Tabernero J, Lakomy R, Prenen H, Prausová J, et al. (2012) Addition of aflibercept to fluorouracil, leucovorin, and irinotecan improves survival in a phase III randomized trial in patients with metastatic colorectal cancer previously treated with an oxaliplatin-based regimen. Journal of Clinical Oncology 30: 3499-3506. [Crossref]

4. Choi YI, Lee SH, Ahn BK, Baek SU, Park SJ, et al. (2008) Intestinal perforation in colorectal cancers treated with bevacizumab (Avastin). Cancer Research and Treatment 40: 33-35. [Crossref]

5. Van Hooft JE, Van Halsema EE, Vanbiervliet G, Beets-Tan RG, DeWitt JM, et al. (2014) Self-expandable metal stents for obstructing colonic and extracolonic cancer: European Society of Gastrointestinal Endoscopy (ESGE) Clinical Guideline. Gastrointestinal Endoscopy 46: 990-1053. [Crossref]

6. Messersmith WA (2019) NCCN guidelines updates: management of metastatic colorectal cancer. Journal of the National Comprehensive Cancer Network 17: 599601. [Crossref]

7. Elice F, Rodeghiero F (2012) Side effects of anti-angiogenic drugs. Thrombosis Research 129: 50-53. [Crossref]

8. Tytgat GN (2007) Hyoscine butylbromide. Drugs 67: 1343-1357. [Crossref]

Copyright: (C2021 Takahashi Y. This is an open-access article distributed under the terms of the Creative Commons Attribution License, which permits unrestricted use, distribution, and reproduction in any medium, provided the original author and source are credited. 